\title{
Base économique et urbanisme dans la ville de Valence (1850-
} 1950) (Espagne)

Roland Courtot

\section{Citer ce document / Cite this document :}

Courtot Roland. Base économique et urbanisme dans la ville de Valence (1850-1950) (Espagne). In: Cahiers de la Méditerranée, $\mathrm{n}^{\circ} 51,1,1995$. Villes intermédiaires en Méditerranée. Tome 2. pp. 101-111;

doi : 10.3406/camed.1995.1150

http://www.persee.fr/doc/camed_0395-9317_1995_num_51_1_1150

Document généré le 31/05/2016 


\title{
BASE ÉCONOMIQUE ET URBANISME DANS LA VILLE DE VALENCE (1850-1950) (Espagne)
}

\author{
Roland COURTOT \\ Université de Provence, centre d'Aix
}

La ville "intermédiaire" est située dans le double sens de la base économique et de la morphologie spatiale :

- au plan du système économique qui fonde ses fonctions de base, la ville intermédiaire est inscrite dans une hiérarchie de relations économiques dont le système urbain ne maîtrise pas le jeu. Les classes sociales urbaines dominantes dans la ville ne sont pas dominantes dans les rapports économiques qu'elles entretiennent avec l'extérieur. Elles sont en situation "dépendante", tant au plan des investissements que des mécanismes du marché.

Au plan des formes de l'espace urbain, la ville intermédiaire n'a pas la complexité d'une métropole où fonctions et classes sociales perdent une partie de leur lisibilité. Mais, comparativement aux petites villes, elle a une "masse critique" qui permet l'inscription dans les formes urbaines de systèmes socioéconomiques clairement désignés par des évolutions de type cyclique caractéristiques des espaces économiques méditerranéens.

La ville de Valence, étudiée à l'occasion d'une recherche de thèse en géographie régionale (COURTOT, 1989), est un observatoire privilégié de cette relation dialectique entre base économique et formes urbaines, dans la mesure où elle a connu, à la fin du XIXème siècle et au début du XXème siècle, une croissance économique et démographique liée en grande partie au développement du système de l'oranger dans les huertas de sa province.

\section{I - VILLE ET AGRICULTURE IRRIGUEE D'EXPORTATION}

Dans la deuxième moitié du XIXème siècle, la base économique de la ville de Valence évolue: d'une ancienne capitale historique, centre d'une huerta irriguée et d'une activité artisanale et manufacturière, on passe progressivement à la capitale d'une région d'agriculture d'exportation. Cette situation n'est pas nouvelle (PIQUERAS, 1989), mais ce qui est nouveau, c'est qu'un système socio-économique se met progressivement en place, qui va dominer la société urbaine par l'importance des relations intersectorielles qu'il engendre et du mouvement économique qu'il soutient. I établit des liens serrés entre la société urbaine et l'agriculture des plaines irriguées environnantes, par le biais de l'appropriation foncière et de l'exploitation directe absentéiste, de l'industrie (qui fournit les produits destinés à la production agricole et ceux destinés à la valorisation commerciale de cette production), du commerce, des transports, des services. 


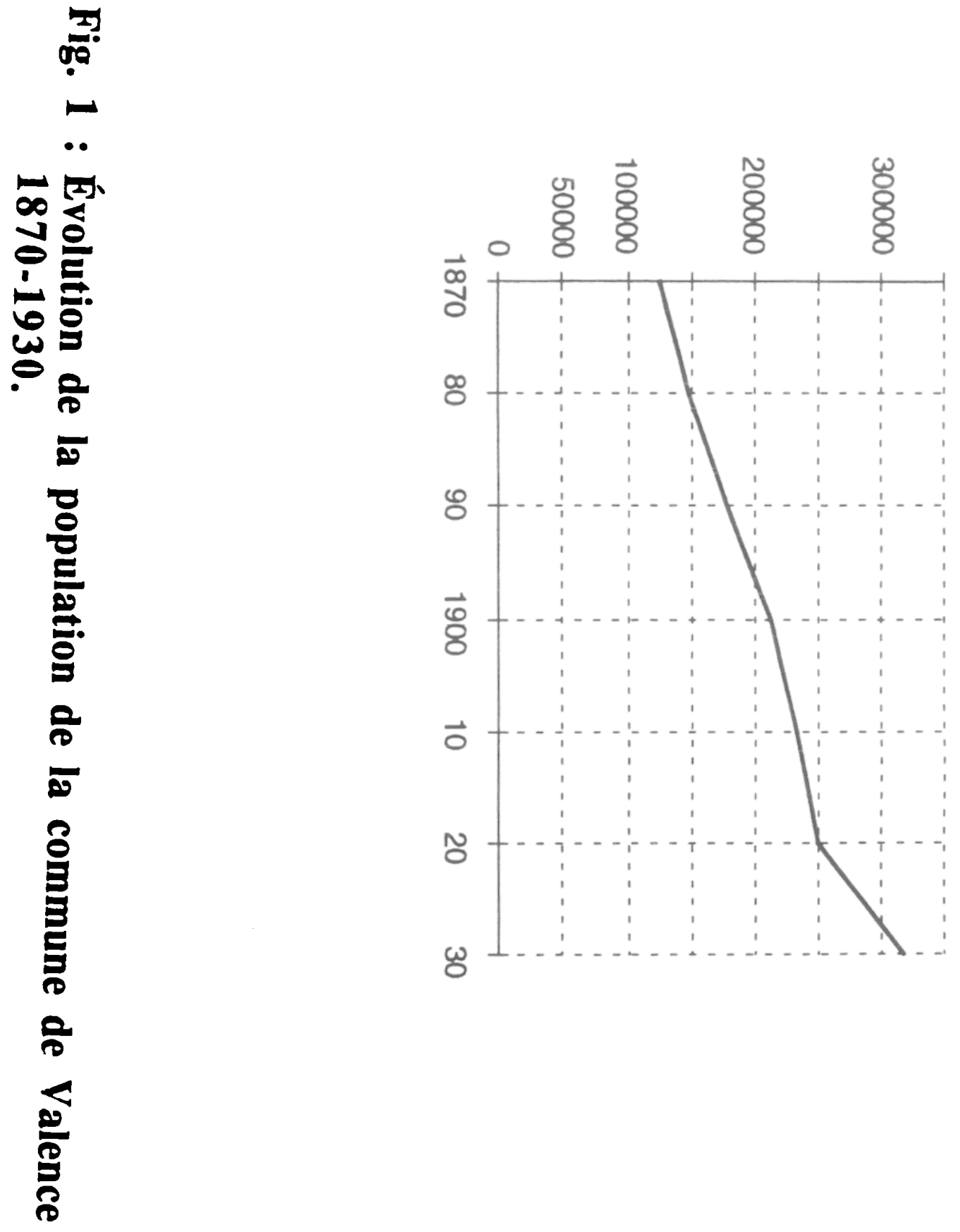


Les activités associées au mouvement portuaire sont en pleine croissance: compagnies de navigation, chantiers navals, industries mécaniques. L'élément principal de ce système est l'orange, dont Valence devient le premier centre d'exportation méditerranéen vers les pays de l'Europe du nord : mais elle n'est pas seule, les oignons, le vin accompagnent ce mouvement.

Cette croissance économique a fait l'objet de nombreuses études et les courbes évolutives coïncident pour dessiner deux phases de croissance économique et urbaine, l'une à la fin du XIXème siècle et l'autre dans les années 1920 (fig. 1), séparées par la situation particulière de la première guerre mondiale, qui conjugue une crise de l'agriculture d'exportation et une croissance de l'activité industrielle.

\title{
II - UNE BOURGEOISIE NOUVELLE DÉPENDANTE
}

Nous avons montré par ailleurs que le système oranger de cette période présente des caractères différents de ceux qu'il acquèrera après la seconde guerre mondiale (fig. 2). C'est alors un système économique fortement dépendant de l'extérieur, en particulier des maisons de commerce anglaises, qui contribuent à l'organisation de la société valencienne liée à l'orange en cercles concentriques (BELLVER MUSTIELES, 1933) :

\begin{abstract}
Au centre, le petit groupe des "vaporistas", représentants des compagnies anglaises et chargés de réunir les cargaisons, qui fournissent pas anticipation de l' argent aux exportateurs valenciens. Ceux-ci, qui forment le deuxieme cercle,...sont chargés $d^{\prime}$ acheter les oranges, de les faire emballer et de les amener d quai en fonction des rotations des navires. Ils font donc travailler les confectionneurs et toutes les autres activités liées d la production et d l'expédition des agrumes, qui forment ainsi le troisième cercle (COURTOT, 1989).
\end{abstract}

Il ne s'agit pas de tout le commerce des agrumes, car les relations avec le marché français sont organisées selon un autre schéma, ni de toute la bourgeoisie valencienne, puisque l'industrie et le commerce ne sont pas exclusivement liés à ce produit. Mais on peut prendre le système oranger comme représentatif de la société et de l'économie valencienne, car il en met en valeur les caractère sociaux principaux :

- la dépendance vis-à-vis de centres d'investissement et de décision extérieurs (les maisons de commerce étrangères qui avancent les financement et les grands ports européens où est fixé le prix de l'orange, Londres, Anvers, Hambourg...) ;

- le "succursalisme" vis-à-vis du gouvernement madrilène, auquel le lobby oranger demande soutien politique et subsides chaque fois que la situation des agrumes se dégrade sur les marchés extérieurs, et qu'elle se répercute sur l'activité et l'emploi des zones de production orangères. On peut 


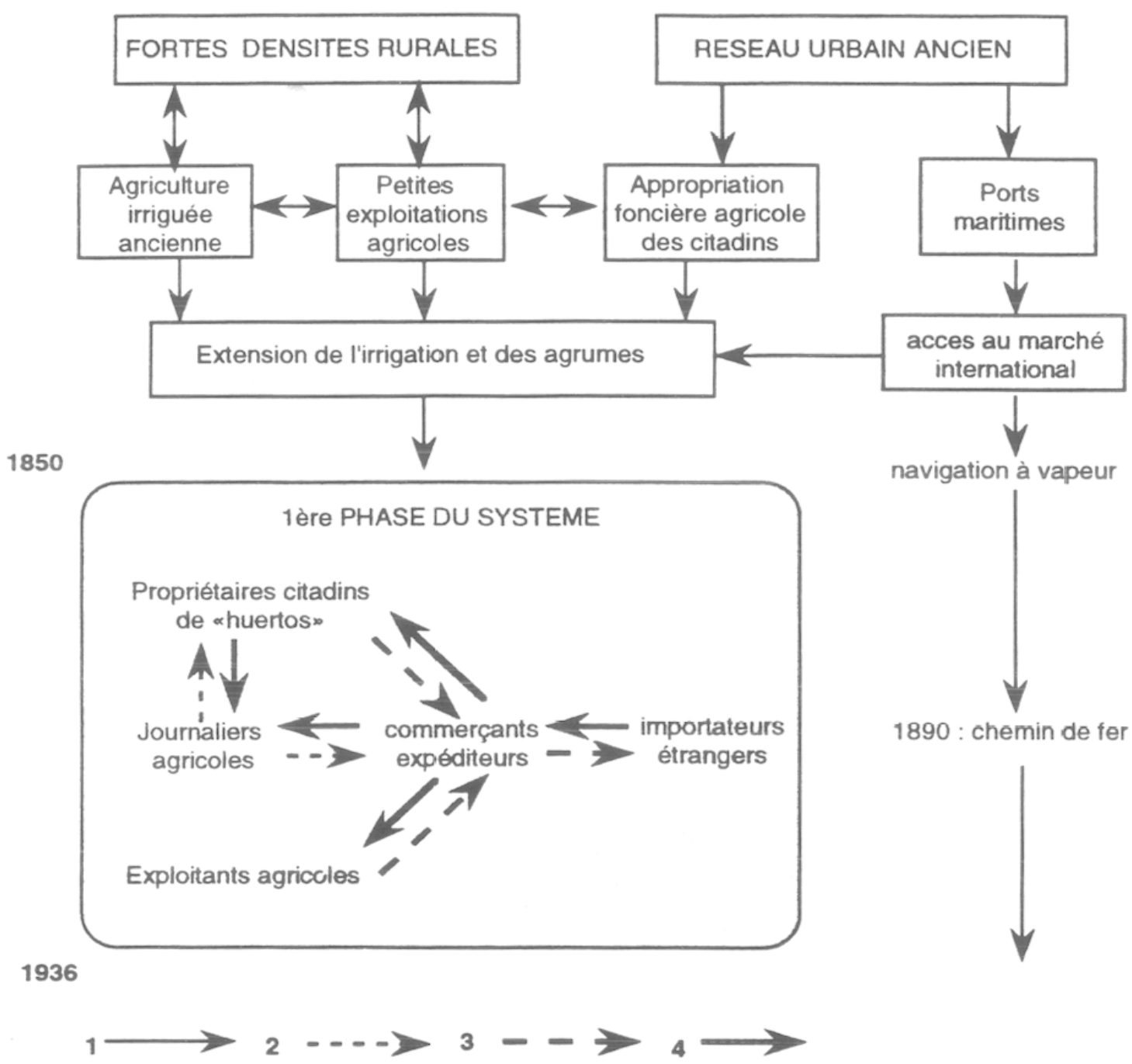

Fig. 2 : Le "système" oranger valencien : première phase, de 1850 à 1936.

- 1 : relation, - 2 : flux de travail, $-3:$ flux de produit, $-4:$ flux monétaire 
donc reprendre à notre compte l'expression fondée par J. FUSTER pour définir en général la position politique et culturelle des Valenciens vis-à-vis de l'État espagnol (FUSTER, 1967), car ce type de comportement apparaît souvent dans l' "Histoire de l'orange" (ABAD GARCIA, 1984).

Les classes sociales dominantes dans la ville de Valence sont donc fortement influencées par et liées à l'évolution de l'économie des agrumes, et leur gestion urbaine est à la fois prolixe dans ses projets et timorée dans ses réalisations. On peut y distinguer schématiquement deux étapes :

- celle d'avant 1914, qui est le fruit de la croissance économique du début de l'ère des agrumes, et qui correspond à l'introduction des grandes innovations urbaines liées à la révolution industrielle (éclairage au gaz, chemin de fer et tramway électrique, assainissement...). C'est le moment où la municipalité est dominée par la bourgeoisie terrienne spéculative qui a pris la place de l'ancienne aristocratie foncière de rente et par les nouveaux entrepreneurs financiers et industriels liés à l'activité portuaire. L'Exposition régionale de Valence en 1909, transformée en exposition nationale l'année suivante, en est à la fois la "vitrine" et l'expression la mieux caractérisée.

- celle de l'entre deux-guerres, où une bourgeoisie moyenne de "cols blancs" s'ajoute à la première en se développant sur les activités tertiaires, commerciales et financières de la ville.

\section{III - LA RENOVATION URBAINE}

Dans la seconde moitié du XIXème siècle, deux conséquences de ces mutations économiques apparaissent dans la morphologie urbaine, en relation avec des opérations d'urbanisme menées par la municipalité (fig.3) :

- dans le centre historique, on assiste à un glissement des lieux de pouvoir qui abandonnent en partie les espaces proches de la cathédrale et du palais du Bayle (l'Audiencia). L'ancienne aristocratie foncière, rentière du sol, qui est installée dans les hôtels anciens du district de la Xerea (rue Caballeros, Trinquete de Caballeros, Navellos, Mar) est en train de céder la place à la nouvelle bourgeoisie de la Restauration et de la Désamortisation qui s'installe dans l'ancien quartier des pêcheurs transformé en un quartier de résidence et de bureaux entre 1870 et 1900 . La rue Barcas devient alors l'axe de ce qui reste encore aujourd'hui le centre des affaires de la ville en accueillant les premiers établissements bancaires.

En même temps une percée est aménagée dans le tissu urbain ancien, parallèlement à la calle del Mar : la nue Paz (1868-1900) :

Pendant le siecle passé et le début de celui-ci, (la rue Paz) a été un foyer de vie sociale et le lieu de résidence de familles significatives, situation qui se reflete dans deux aspects importants: le commercial et l'architectural (TEIXIDOR, 1971). 


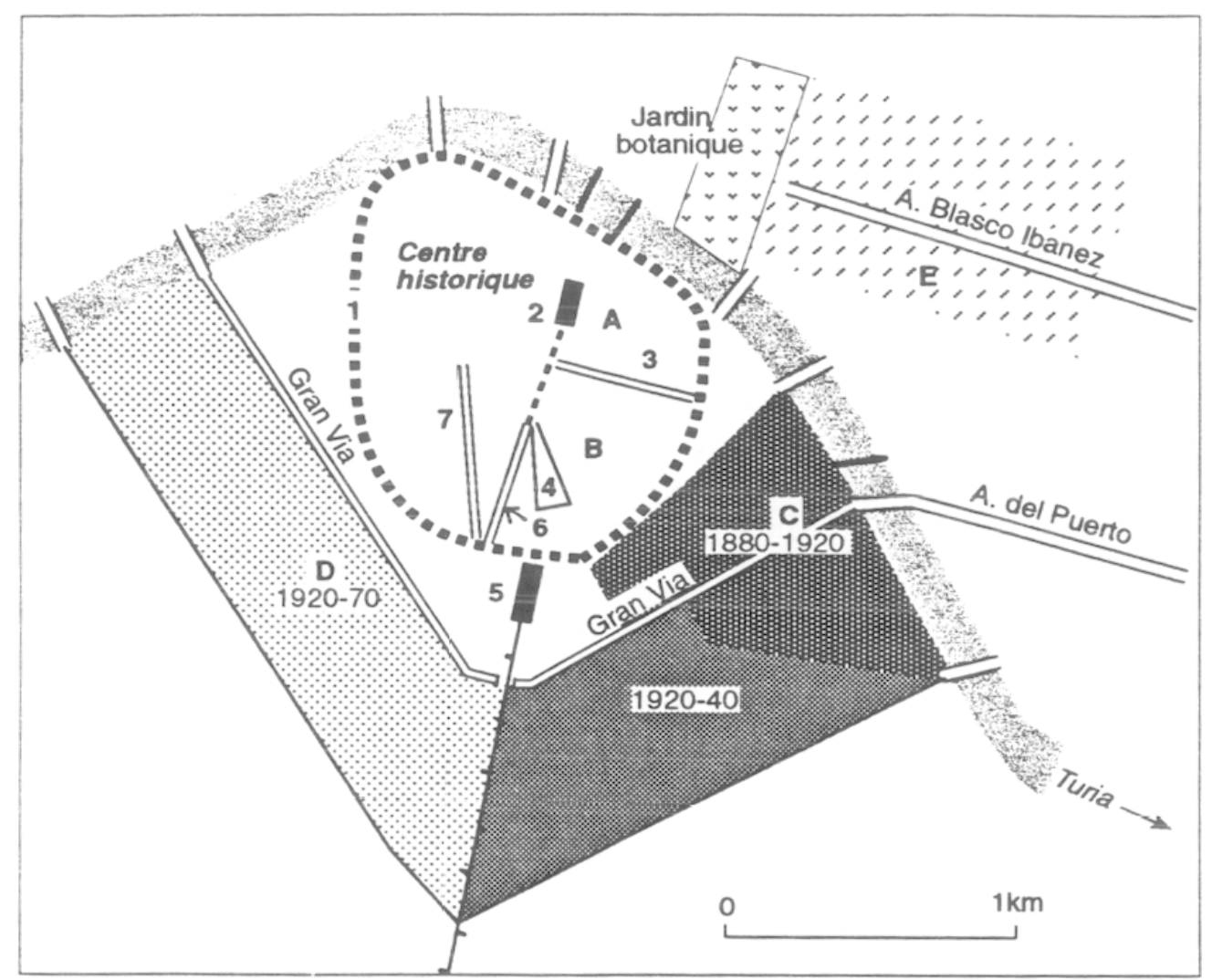

\section{Fig. 3 : Localisation des projets et opérations d'urbanisme à} Valence : 1850-1950.

- 1 : avenues sur le tracé des anciens remparts, - 2 : cathédrale, - 3 : percée de la rue Paz, - 4 : place centrale, - 5 : gare du Nord, - 6 : rue San Vicent, - 7 : percée de l'avenue Baron de Carcer (del Oeste) (les grands axes sont indiqués dans leur situation actuelle) A : quartier aristocratique ancien, $B$ : centre des affaires, $C$ : “ensanche" bourgeois, D : "ensanche" ouvrier, E : cité-jardin (paseo de Valencia al Mar). 
L'installation de la première gare-terminus et de la mairie sur les autres côtés de la place de San Francesc, en face du barrio del Mar rénové préfigure la fonction centrale que cette place ne cessera plus de jouer (aujourd'hui place du Pays valencien, après avoir été place du Caudillo de 1939 à 1975)

- à l'extérieur des remparts :

Dès le milieu du XIXème siècle, dans la fièvre des innovations industrielles et urbaines (le chemin de fer Valencia-Jativa est terminé en 1842), un premier projet d'extension urbaine voit le jour (1858). Mais il faut attendre le début de la destruction des murailles (1865) pour qu'un nouveau plan d'extension soit approuvé en 1887, qui , à l'instar du plan Cerdà à Barcelone, organise une croissance "quadrillée" de la ville au-delà de la ceinture des nouveaux boulevards apparus avec la chute des murailles (avenidas).

Il s'intéresse d'abord à l'espace compris entre le chemin de fer et le fleuve, pla del Remedio, qui se trouve en continuité, au delà de la calle Colon, avec le nouveau "centre" de la ville. C'est l'ensanche par antonomase, et celui qui se construit et se "remplit" le plus rapidement entre 1887 et 1914. La gran via qui en est l'axe cardinal porte d'ailleurs le nom, et la statue, du Marqués del Turia : il s'agit de J. Campo i Pérez (1814-1899), Marqués de Campo, Maire de Valence, député, sénateur et chef de file du capitalisme financierterritorial-commercial-urbain et ferroviaire de la ville dans la seconde moitié du XIXème siècle (LLUCH, 1976, p. 124).

La bourgeoisie de l'orange, formée de tout ce qui gravite autour du commerce d'exportation, expéditeurs, exportateurs, consignataires, transporteurs, propriétaires fonciers, etc..., s'y installe et affiche sa prospérité dans une architecture qui suit les modèles européens, en particulier le langage "moderniste".

Une étude portant sur la résidence des grands propriétaires fonciers d'agrumes résidant en ville en 1972 montre d'ailleurs une grande permanence de ces localisations urbaines : la majeure partie de ceux-ci se trouve dans le quartier des affaires et dans "l'ensanche" Sud-Est, qui porte encore aujourd'hui le nom d'eixampla par antonomase (COURTOT, 1990).

\section{IV - LA PÉRIODE DE L'ENTRE-DEUX-GUERRES : ÂGE D'OR DU COMMERCE DES AGRUMES}

La première guerre mondiale marque un ralentissement de l'activité économique de la région valencienne, car l'exportation des agrumes s'effondre, d'abord par suite de l'économie de guerre des puissances belligérantes, puis par suite du blocus maritime et de la guerre sous-marine décrétée par l'empire allemand. Si l'activité industrielle en tire profit, le retour de la paix ramène au premier plan le système oranger, qui prospère jusqu'à la crise de 1929. 


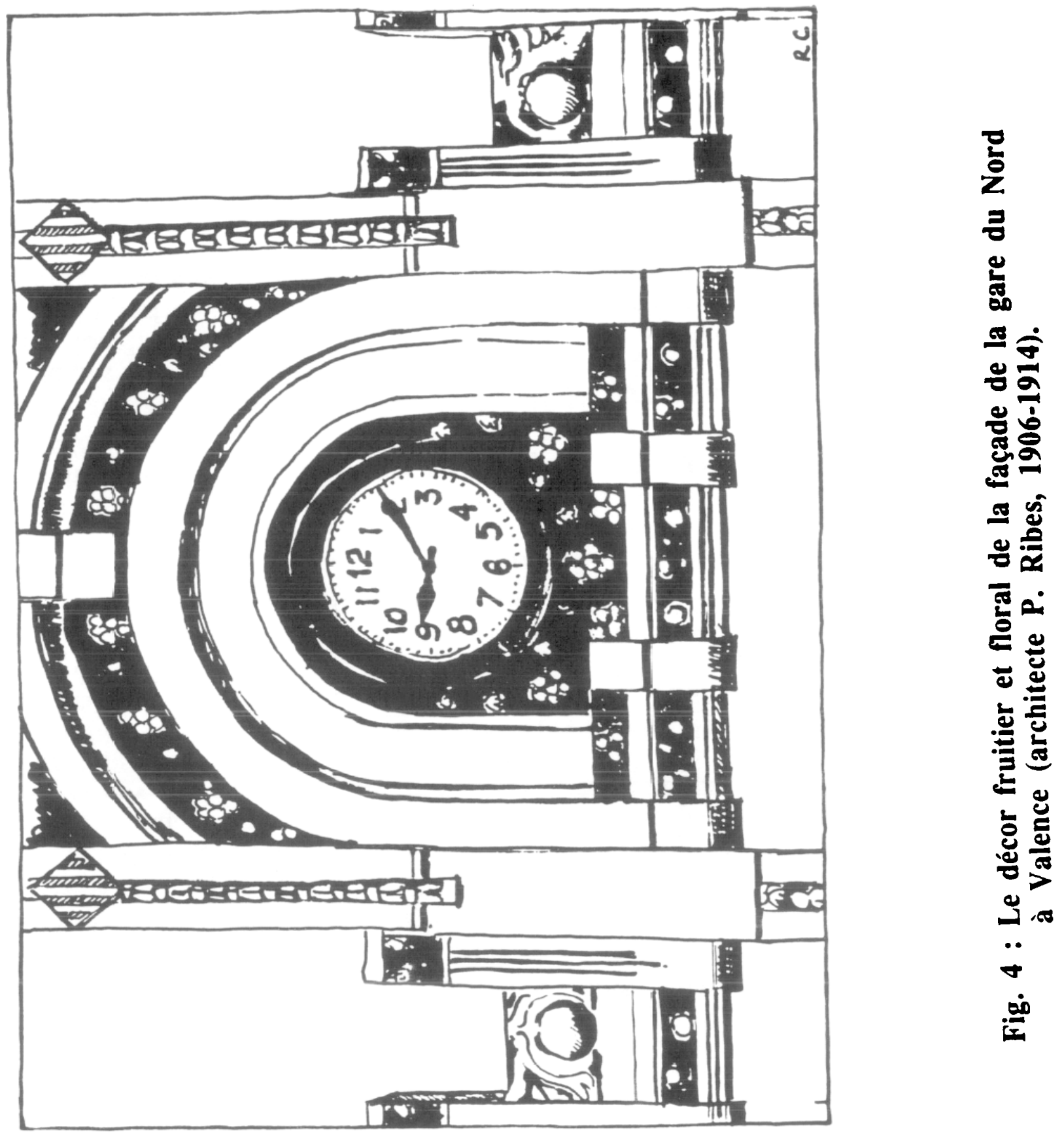


C'est alors pour la ville une période de réalisations architecturales qui complètent les transformations en cours depuis la fin du siècle précédent : la nouvelle gare de chemin de fer, ramenée au-delà des avenues périphériques affirme maintenant dans sa décoration l'importance des agrumes dans la vie économique valencienne (fig. 4). Sur la place centrale (alors "d'Emilio Castelar"), la Mairie, la Poste, magnifient dans leurs façades décorées ce style qu'on appellera le néobarroque valencien. Le quartier de San Francesc renforce ses fonctions centrales avec l'installation de nouveaux édifices bancaires, en particulier celui du Banco de Valencia en 1927.

A partir de 1930 la partie nord de la place centrale est élargie et son pourtour se couvre, comme la rue San Vicent qui la recoupe, de grands immeubles de bureaux (en particulier celui qui abrite l'Ateneo Mercantil) où se mêlent dans l'architecture les styles néobarroque, castissiste et rationaliste, mélangeant modernité et tradition (projet de l'architecte Goerlich en 1929).

En même temps, dans la partie moderne de la ville, le plan d'extension de 1907, qui avait complété celui de 1887 en l'étendant à l'ouest, se remplit progressivement, toujours plus rapidement dans son versant oriental "bourgeois" que dans son versant sud et occidental, destiné à une population plus modeste :

En général il s'agit de constructions de caractère spéculatif, destinées do la résidence de la petite bourgeoisie ou du prolétariat (LLORENS, 1974).

L'ensanche a même sauté la barrière du fleuve et les premiers alignements d'un quadrillage parallèle à l'avenida del puerto, apparaissent sur le llano del Real, au delà de la Alameda, promenade de rive gauche du fleuve. Le projet architectural est ici celui d'une cité jardin dont l'axe principal serait un paseo de Valencia al mar, qui s'ouvrirait une voie triomphale de plusieurs kilomètres du jardin botanique à la plage de la Malvarossa.

\section{CONCLUSION}

On pourrait voir, dans ce foisonnement (relatif) de projets et de plans d'urbanisme, une grande dynamique des classes sociales dirigeantes appliquée à la ville dont elles détiennent les pouvoirs municipaux. Il faut en fait tempérer cette conclusion apparente de deux façons.

La première nous est fourni pas les historiens de l'architecture : les modèles que les architectes valenciens appliquent sont bien ceux des grands mouvements européens (art nouveau avant 1914, puis art déco et rationalisme dans l'entre-deux-guerres) mais ils les détournent souvent au profit des modèles traditionnels du néobarroque et du casticisme, ce qui fait dire à $T$. LLORENS qu'il n'y a pas eu d'avant-guarde architecturale à Valence et que cela est à mettre en relation avec : 
la structure sociale générale du Pays (valencien) et la mentalité conséquente de sa classe dirigeante (LLORENS, 1974, p.88).

La seconde apparaît lorsqu'on considère l'écart qui existe, dans le temps et dans l'espace, entre les projets et leur réalisation. A l'intérieur du centre historique, si la percée de la rue Paz est rapidement menée à bien, celle de l'avenue de l'Ouest, projetée en 1910, reprise dans le plan de 1929, ne sera finalement réalisée qu'à partir de 1940, et encore partiellement, puisqu'elle s'arrête en 1965, derrière le marché central, en ayant parcouru moins de la moitié du trajet qui devait lui permettre de traverser de part en part le vieux centre du sud au nord, jusqu'au fleuve (aujourd'hui avenue "Baron de Carcer").

Le seul projet d'extension mené à bien de façon à peu près complète dans la période étudiée est celui du sud-est, de l'ensanche burgés. L'ensanche de l'ouest, planifié dès 1907 , ne sera vraiment "rempli" qu'après 1940 , et sans imprimer un quadrillage parfait au tissu antérieur des chemins et voie radiales divergeant de la ville. La "cité-jardin" (ensanche -parc) de la rive gauche du Turia, connaît un début de réalisation sous la République (1930-36) mais ce n'est que dans la période de forte croissance urbaine postérieure aux années cinquante, que cet espace est uccupé par des immeubles collectifs et de grands services publics (université); on est loin de la cité-jardin, et le paseo de Valencia al Mar, qui a pris le nom d'avenida Blasco Ibañes, n'arrive toujours pas à la mer.

Mais ne faut-il pas y voir aussi, au-delà d'un comportement social conservateur et spéculatif de la part des classes sociales dirigeantes, les conséquences des à-coups de l'économie agrumicole qui rythment l'évolution de la base économique de la cité jusqu'au milieu du XXème siècle ? 


\section{BIBLIOGRAPHIE}

ABAD GARCIA V., Historia de la naranja, Comité de la Gestión de Frutos Cítricos, Valencia, 1984, 447 p.

BELLVER MUSTIELES J., Esbozo de la futura economía valenciana, Camara Oficial de Comercio, Industria y Navegacion, Valencia, 1933, 32 p.

COURTOT R., Campagnes et villes dans les huertas valenciennes, Mémoires et documents de géographie, CNRS, Paris, 1989, 191p.

COURTOT R., Les propriétaires fonciers et la ville. Valencia (Espagne), Géographie Sociale, 10, CNRS URA 915 et Publications de l'Université de Caen, 1990, p.155-163.

FUSTER J., Nosotros los valencianos, colecciones Peninsula, Edicions 62, Barcelona, 1967, $256 \mathrm{p}$.

LLORENS T., El moviment moderne $i$ el racionalisme a l'arquitectura i l'urbanisme valencians, Arguments, l'Estel, 1-1974, p.59-96 1976, $254 \mathrm{p}$.

LLUCH E., La via valenciana, Collection 3 i 4, E. Climent, Valencia,

PIQUERAS J., La agricultura valenciana de exportación y su formación histórica, Instituto de Estudios Agrarios, Pesqueros y Alimentarios, Serie Estudios, Madrid, 1985, 249 p.

SIMO T., La arquitectura de la renovación urbana en Valencia, Albatros Ed., Valencia, 1973, 274 p.

TEIXIDOR M.J., La calle de la Paz (Valencia), Cuadernos de Geografia, Universidad de Valencia, $\mathrm{n}^{\circ}$ 8, 1971, p. 83.

TEIXIDOR M.J., Valencia, la construccio d'una ciutat, Institucio Alfons el Magnanim, Valencia, 1982. 